\title{
Design Principles for Tactile Interaction
}

\author{
B. P. Challis \& A. D. N. Edwards \\ Department of Computer Science \\ University of York \\ York, YO10 5DD, UK \\ bpc@cs.york.ac.uk \\ alistair@cs.york.ac.uk
}

\begin{abstract}
A preliminary set of principles for tactile interface design are described. These have been constructed using the findings of a study into the presentation of music notation to blind people.
\end{abstract}

\section{Keywords}

Tactile, haptic, design principles, overlay.

\section{INTRODUCTION}

Although we rely on touch to perform many everyday actions, the real potential for enhancing such interaction is often neglected and, where tactile feedback is available, it is often on an ad hoc basis. Tactile interaction could benefit many computer based applications, whether on a standalone basis or in support of visual and auditory interaction. However, if this is to become a reality, then solid design principles will need to be established such that efficient design strategies can be realised. The work presented here is a first-step towards achieving this target. Using existing psychological knowledge along with results from studies into computer-based tactile interaction a number of foundation principles are outlined.

\section{BACKGROUND}

There are many reasons why users could benefit from the inclusion of haptic interaction within a given system. Visually dominated interfaces are commonplace yet may not always be the most efficient or intuitive method for performing a given task. In the most extreme scenario the graphical user interface simply excludes visually impaired users. However, there are also instances where a user needs to control and observe a process where the process itself is already providing visual feedback (e.g. slide projector, radio controlled equipment, stage lighting etc.). In these circumstances, the user is likely to benefit from some level of increased haptic feedback so that their visual attention can be maximised in terms of directly observing any changes being made. In certain other circumstances, using a car radio whilst driving for example, a strategic shift in balance from visual toward tactile interaction could prove to be safer.

Given the amount of information that is sometimes presented within graphic displays, it could be a real asset to incorporate alternative display methods to help reduce potential confusion. Besides helping to alleviate the growing problem of overcrowded, and therefore, confusing graphic displays, haptic displays present possibilities for providing physical manipulation of controls in a more intuitive fashion. Many music applications attempt to emulate the environment that the user is likely to be familiar with by providing graphic faders, rotary knobs and push buttons. Although these look like controls that might be found on a mixing desk or synthesiser, the precision in use that is expected is often lost when trying to manipulate these graphic images using a mouse and keyboard.

The increasing availability of new force-feedback devices presents many possibilities for creating 'virtual' displays which could benefit some of the previous examples. However, similar solutions could be achieved using dedicated static displays and there is therefore a trade-off to be made according to which display approach is adopted. With virtual displays, the technology used allows the display to be instantly updated or completely altered to meet new requirements within a task. Whilst this is an obvious asset from the perspective of flexibility, this exploration method cannot provide the user with the finer levels of tactile feedback. Such feedback allows us, for example, to discriminate between many types of fine textures and tactile patterns, identify and discriminate between raised symbols and to notice even small changes in height between separate objects. This kind of feedback is available using dedicated static tactile displays although at extra cost in terms of producing each display. It is likely, therefore, that there will be design principles which will be common to both approaches along with additional principles which are specific to each display type.

\section{DESIGN PRINCIPLES}

The focus of this research has been on the use of static displays to enhance tactile interaction within computerbased systems. An example application for the delivery of music notation to blind people has been created [1,2]. The system, called Weasel, uses PVC tactile overlays on an Intellikeys touchpad in conjunction with speech output and audio output. Results obtained from the close observation of users working with the system have been used along with existing psychological knowledge on interaction with raised lines [1,2,9], tactile symbols [3,7,11,12] and textures $[6,8,10]$, to create a set of fundamental design principles for tactile interaction.

Graphical music notation can present a large quantity of information which is perceived by the reader in a near 
parallel fashion. Often, much of this information will be redundant to a particular learning task so the reader simply ignores that data which is unwanted. Non-visual music notations (e.g. braille music and 'Talking Scores') present exactly the same information but in a serial fashion; effectively, the learner must interpret every single instruction before deciding whether it is important to the task in hand.

Besides producing large amounts of translated instructions, these alternative approaches do not assist the reader in forming an adequate mental image of the layout of the musical extract that they are working with. A page of music contains a certain number of lines of music and each of these lines will have a certain number of bars belonging

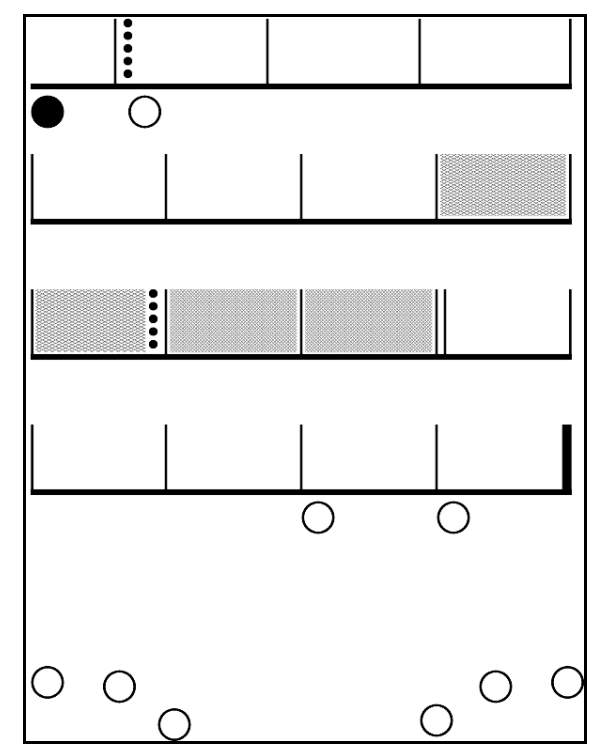

Figure 1. An example of one of the PVC overlays used within the Weasel music notation system.

to it. There may be an incomplete or 'lead-in' bar at the beginning along with indications as to where one section stops and another starts. These sections might need to be repeated and if they do there might be substitute sections (first and second time bars) for each repeat.

All of these elements are invaluable in terms of building an impression of 'shape' which the reader can easily relate to. Without this, the reader is unable to easily communicate to fellow musicians using common terms of reference such as "the third line of the first page" or perhaps "from the third bar of the top line to the second-time bars".

The aim of the Weasel Project has been to address this particular issue by presenting this aspect of music notation as a tactile overlay on a touchpad. The user can quickly gain an impression of the structural layout of a page of music and then interact directly by pressing onto the overlay to retrieve a description of the music within a particular bar. This is delivered as either musical playback or a spoken description using speech synthesis and the reader has further control over what level of detail is presented.

\section{Initial Overlay Design}

Vacuum-formed PVC overlays were used in preference to the more common method of 'swell paper'. Although the former are more complex to manufacture, they can afford considerable differences in height within the same overlay which 'swell paper' cannot. The overlays were designed using a very simple visual-to-tactile mapping such that each overlay looked like its visual counterpart (see Fig. 1). Each 'tactile page' was approximately 'legal-letter' size and was presented in portrait orientation. A $1.5 \mathrm{~mm}$ high guideline was provided beneath each line of bars and different levels of height were used for barlines $(2 \mathrm{~mm})$, repeat marks ( $4 \mathrm{~mm}$ high dots) and the final barline $(4 \mathrm{~mm})$. First-time and second-time bar areas were represented using textures and these were of a lower height than the guideline. Circular symbols were occasionally located just beneath the guideline to represent the presence of either a dynamic or a number of spoken descriptions. These could be pressed to retrieve the description and where more than one item was present the symbol could be double-clicked to progress to the next item in the list.

In addition, there was a control section located at the bottom of the overlay which provided access to a menuing system for changing various settings. This was designed to be controlled using the index, middle and ring finger of each hand where the left hand controlled the selected option and the right hand selected the item within that option.

\section{Foundation Design Principles}

In the initial design stage of the project, the following principles were employed:

A consistency of mapping should be maintained such that descriptions of actions remain valid in both the visual and the non-visual representations.

An example in music would be a reference to a location such as "The last bar of line two on page three". The same would apply to the relative location of on-screen controls including the directions in which they can be moved.

\section{The tactile representation within an interface should focus on data that is static.}

This was partially due to the lack of dynamic displays that can function at a tactile level. However, even if a display was dynamic there would still be a problem in notifying the user exactly where within the display a change had taken place. Reliance on visual feedback would be defeating the purpose of integrating tactile interaction in the first place.

\section{Height should be used as a filtering mechanism.}

The user should be able to home in on certain information types using height as a discriminating feature. 


\section{User Testing}

The Weasel system has been assessed by a group of six users. All of the users were competent musicians who also possessed a good knowledge of the main concepts of music notation. Five of the group were sighted and were therefore blindfolded whilst using the system and the sixth member was blind.

The group was first trained to use the system using a series of five overlays which gradually introduced the various tactile components used. After this training period, each user was asked to perform a number of tasks using two completely new overlays which included all of the tactile components used previously.

The testing was in two parts. Firstly, the user was asked to explore the new overlay and systematically describe each tactile object and its meaning as they located it. After this, the user was asked to perform a number of tasks each of which involved changing various settings and then moving to specific locations within the music and retrieving a description of that particular area. Although the results of these tests were quantifiable, observation was regarded as being just as valuable from the perspective of understanding how or why certain actions might be complex or confusing to the user.

\section{Results}

The results from the testing showed that the users were capable of understanding, navigating around and interacting with the overlays. However, through general observation and comments that were made by users, there were obvious problem areas within the interface. It quickly became apparent that the simple mapping used had led to the inclusion of quite large uninformative areas. These were such that users often seemed confused as to their whereabouts within the overlay. In addition, some of the users performed a task incorrectly because they had failed to locate the topmost line of music, their actions were, however, accurate within the context of that line.

Users also exhibited problems with double-clicking which appeared to produce quite clumsy actions; this observation was reinforced from general comments on the awkward nature of performing a double-click with virtually no haptic feedback. The guideline was not as useful in establishing an exploration strategy as had been hoped. This was, again, probably partly due to the visual-to-tactile mapping that was adopted which meant that even though a guideline was available it was still necessary for the user to have to leave this to explore other areas.

These results have led to the expansion of the original three foundation design principles to now include the following additional principles:

Good design will avoid an excess of 'empty space' as this is a significant source of confusion.
The term 'empty space' is used in reference to areas on a display that do not communicate anything useful to the user. If a user can place a fingertip into a display without quickly locating a feature that gives them a meaningful cue they are effectively in 'empty space'. It might not be possible to eradicate this but it should be minimised.

\section{A simple visual-to-tactile mapping is likely to produce many problems and is therefore unlikely to be the most efficient design strategy.}

This is not in conflict with the first principle that was described. A consistency of mapping can and should be maintained but the likelihood is that the tactile display will not actually look like its visual counterpart.

Good design practice should, whenever possible, encourage a specific strategy for the exploration of a particular display.

If the display is to be used in a non-visual way then this principle becomes particularly significant. However, even when used in support to a visual display this principle remains valid. It would be undesirable for the user to have to visually monitor the tactile display to observe their progress within an action.

\section{Double-clicking is an inappropriate form of interaction within static displays.}

Without haptic feedback, double-clicking can quickly becomes inefficient leading to the user perceiving closure when it has not been achieved. Alternative methods using multiple points of contact and timed single-presses are being explored as part of the Weasel project.

\section{A display should be sized and orientated such that users are not expected to overreach to discover the full extent of the display.}

This may seem obvious but it is surprising how often users will fail to fully explore a display when they are unable to see their progress. A suitable maximum display area is approximately A4 sized in landscape orientation.

\section{Tactile objects should be simple.}

When designing objects for use within a graphic display it is possible to employ a considerable number of dimensions by which differences can be achieved. Tactile interaction can allow subtle changes within a dimension e.g. changes in height, width or texture. However, the greater the number of dimensions along which the user is expected to notice change, the more complex the object will appear to be to the user. Changes along less dimensions will make for a more immediately recognisable object which will in turn provide a basis for faster and more accurate interaction.

\section{FUTURE WORK}

A new design of the Weasel system is currently being implemented which is based around the extended set of design principles. 'Empty space' is being reduced to being no greater than the approximate size of a fingertip and this 
is being used to also provide a more efficient and intuitive strategy for exploration. The new overlays (see Fig. 2) are still constructed from PVC but are now presented in landscape orientation. The bar-areas and guideline are now integrated into a single strip approximately $15 \mathrm{~mm}$ wide and $300 \mathrm{~mm}$ long. This approach presents the user with a simple left-to-right reading strategy which will help minimise the level of exploration that is required within the overall display. The height of a normal bar-area is approximately the thickness of standard printing paper. Barlines are about the width of a fingertip and approximately $1 \mathrm{~mm}$ high. Repeat marks are presented as 'ramps' that rise from within the bar-area up to a higher barline of $2 \mathrm{~mm}$.

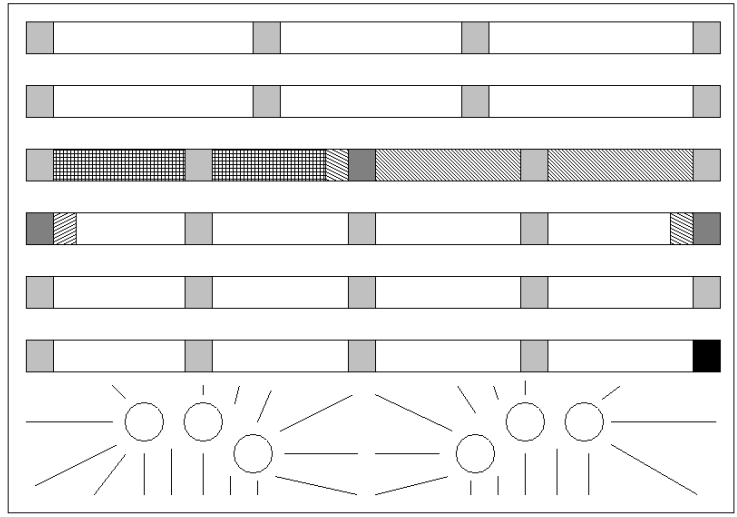

Figure 2. An example of one of the new

Weasel overlays.

The controls for the menuing system have not been changed as these appear to have functioned quite satisfactorily. However, the lists of options and items now 'wrap-around' rather than terminating at two extremes. Alternative methods to double-clicking are being explored within the new design. One such possibility is for the user to press and hold an 'active' area and after a short period another action (e.g. progressing through a list) will be activated automatically.

\section{CONCLUSION}

Tactile interaction is often overlooked within interface design even though it could prove to be a more appropriate approach to adopt for certain circumstances. Although tactile interaction is not unusual within many everyday actions it is still relatively novel and perhaps somewhat underused within the human-computer interface. Successful integration within computer-based systems is only likely to be achieved if an effective design strategy can be employed. It is hoped that the continued development of the foundation design principles, as presented here, will form an effective basis for interface designers to begin to maximise the potential for tactile interaction within their applications.

\section{ACKNOWLEDGEMENTS}

This research has been funded by the Engineering and Physical Sciences Research Council (award ref. 96309035). We would like to thank Intellitools Inc. (55
Leveroni Court, Suite \#9, Novata, CA. 94949) for providing an Intellikeys touchpad to help with the practical aspects of this research.

\section{REFERENCES}

1. Bentzen, Billie Louise and Peck, Alec F (1979). Factors affecting the traceability of lines for tactile graphics. The Journal of Visual Impairment and Blindness. 73(7), 264269.

2. Berla, Edward P and Murr, Marvin J (1975). Psychophysical functions for the active tactual discrimination of line width by blind children. Perception \& Psychophysics. 17(6), 607-612.

3. Berla, Edward P and Butterfield, Lawrence H (1977). Tactual distinctive features analysis: Training blind students in shape recognition and in locating shapes on maps. The Journal of Special Education. 11(3), 335-346.

4. Challis, B. P and Edwards, A. D. N. (2000). Weasel: A system for the Non-visual Presentation of Music Notation. Proceedings of ICCHP 2000, 113-120.

5. Challis, B. P and Edwards, A. D. N. (2000). Design Principles for Non-visual Human-Computer Interaction. ACM SIGCAPH Bulletin, (Spring).

6. James, G A and Gill, J M (1975). A pilot study on the discriminability of tactile areal and line symbols for the blind. American Federation for the Blind Research Bulletin. 29,

7. Lambert, L M and Lederman, S J (1989). An evaluation of the legibility and meaningfulness of potential map symbols. The Journal of Visual Impairment and Blindness. October, 397-403.

8. Lederman, Susan J and Kinch, Denise H (1979). Texture in tactual maps and graphics for the visually handicapped. The Journal of Visual Impairment and Blindness. 73(6), 217-227.

9. Lederman, Susan and Campbell, Jamie I (1983). Tangible line graphs: An evaluation and some systematic strategies for exploration. The Journal of Visual Impairment and Blindness. 77(4), 108-112.

10. Millar, Susanna (1985). The perception of complex patterns by touch. Perception. (14), 293-303.

11. Nolan, C Y and Morris, J E (1971). Improvement of tactual symbols for blind children: Final report. American Printing House for the Blind.

12. Simpkins, Katherine E (1979). Tactual discrimination of shapes. The Journal of Visual Impairment and Blindness. 73(3), 93-101. 